\title{
Uncoupling of complex regulatory patterning during evolution of larval development in echinoderms
}

\author{
Kristen A Yankura, Megan L Martik, Charlotte K Jennings, Veronica F Hinman*
}

\begin{abstract}
Background: Conservation of orthologous regulatory gene expression domains, especially along the neuroectodermal anterior-posterior axis, in animals as disparate as flies and vertebrates suggests that common patterning mechanisms have been conserved since the base of Bilateria. The homology of axial patterning is far less clear for the many marine animals that undergo a radical transformation in body plan during metamorphosis. The embryos of these animals are microscopic, feeding within the plankton until they metamorphose into their adult forms.

Results: We describe here the localization of 14 transcription factors within the ectoderm during early embryogenesis in Patiria miniata, a sea star with an indirectly developing planktonic bipinnaria larva. We find that the animal-vegetal axis of this very simple embryo is surprisingly well patterned. Furthermore, the patterning that we observe throughout the ectoderm generally corresponds to that of "head/anterior brain" patterning known for hemichordates and vertebrates, which share a common ancestor with the sea star. While we suggest here that aspects of head/anterior brain patterning are generally conserved, we show that another suite of genes involved in retinal determination is absent from the ectoderm of these echinoderms and instead operates within the mesoderm.

Conclusions: Our findings therefore extend, for the first time, evidence of a conserved axial pattering to echinoderm embryos exhibiting maximal indirect development. The dissociation of head/anterior brain patterning from "retinal specification" in echinoderm blastulae might reflect modular changes to a developmental gene regulatory network within the ectoderm that facilitates the evolution of these microscopic larvae.
\end{abstract}

\section{Background}

The astonishing diversity of animal forms, coupled with the complex life histories typical of many marine invertebrates, presents numerous challenges in inferring the ancestral character of members of the closely related phyla collectively known as the deuterostomes. Modern molecular phylogenies place four phyla within the monophyletic deuterostomes: Echinodermata and Hemichordata comprise a distinct clade called the Ambulacraria [1-3] that is a sister group to Chordata [4]. Xenoturbella is a recent out-group addition to the Ambulacraria [5].

Within the Ambulacraria, the free-swimming, bilaterally symmetric larvae of echinoderms, especially the bipinnaria larva of sea stars and the auricularia larva of

\footnotetext{
* Correspondence: veronica@cmu.edu

Department of Biological Sciences, Carnegie Mellon University, Pittsburgh, PA 15213, USA
}

sea cucumbers, share many similarities with the tornaria larva of indirectly developing hemichordates. These microscopic larvae have an apical concentration of serotonergic neurons [6] and one or two concentrations, or bands, of cilia used to feed and swim in the plankton $[7,8]$. Neurons lie beneath this ciliated epithelium and innervate the bands [9]. Similarities in larval form initially provided the basis for many of the hypotheses surrounding the evolutionary origins of the chordates and, in particular, the centralized nervous system. These hypotheses, in which a microscopic larval stage is assumed ancestral to the entire deuterostome clade, propose that a centralized nervous system evolved from an infolding of the larval ciliary bands [10-12].

Not all Ambulacrarians develop through a larval stage, however, and recent comparisons of regulatory gene expression have revealed that orthologs of many genes

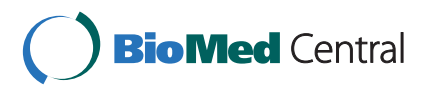


and signaling molecules involved in vertebrate neural patterning are expressed in spatially restricted domains along the anterior-posterior (AP) axis of the direct developing vermiform hemichordate juvenile Saccoglossus kowalevskii $[13,14]$, a species that does not develop via a tornaria larva. This general correspondence in AP position of orthologs between the vertebrate and hemichordate nervous systems implies some homology in axial patterning. In addition, the broad ectodermal expression of these genes in hemichordates suggests that a diffuse panectodermal neural domain was the ancestral state of the deuterostome nervous system and that the centralization event occurred later within the lineage leading to the chordates. These findings and those of other researchers (reviewed in $[15,16]$ ) therefore negate the need to invoke a ciliated ancestor for deuterostomes as suggested by Garstang [10]. In addition, general homologies in axial expression patterns between vertebrates and direct developing protostomes have been observed as well [17], suggesting that common patterning mechanisms have been employed since the radiation of Bilaterians. Indirect developing larval forms have less obviously distinguished body axes and no strong homology of axial patterning and, as a result, appear derived and possibly secondarily simplified in comparison.

Here we examine the expression of regulatory gene orthologs that have known or suspected roles in patterning the axial neuroectoderm of many protostome and deuterostome embryos within the indirectly developing sea star, Patiria miniata (previously Asterina miniata), which forms a typical bipinnaria larva. We show that these genes are expressed in diffuse concentric ectodermal domains that pattern the early embryonic axis. Furthermore, we observe in the sea star a general correspondence of domains of orthologous gene expression to those found along the AP and dorsal-ventral (DV) axis of direct developing deuterostomes. In addition, we detect expression of retinal determining gene orthologs in the mesoderm of echinoderm larvae, but not within the ectoderm of gastrulating embryos. We discuss the role of this implied modularity of regulatory patterning during evolution.

\section{Results and discussion} Isolation of sea star transcription factors

P. miniata orthologs of regulatory genes that have known or suspected roles in patterning the axial neuroectoderm of many protostome and deuterostome embryos were isolated using a candidate gene approach. Recombinants for the following seven genes that encode homeodomain proteins were obtained: retinal homeobox $(r x)$, optix-like homeobox 3 (six3), gastrulation and brain-specific homeobox ( $g b x)$, lim domain homeobox 2 (lhx2) and paired box homeobox 6 (pax6), as well as members of the Nkx gene family, $n k 2.1$ and $n k 1$. We also identified partial sequences of eyes absent (eya), the ets family gene pea3, and two $\mathrm{C} 2 \mathrm{H} 2$ zinc-finger genes, zic and krupple-like factor 13 (klf13). The following four winged-helix forkhead box genes were isolated: foxq2, foxj1, foxd and foxg. A complete list of orthologs, sequence lengths, and orthology of gene sequences is provided in Additional file 1.

\section{Animal-vegetal patterning of the sea star blastula ectoderm}

Sea star late blastulae have a morphologically distinct animal-vegetal (AV) axis that is first readily observed when elongation of cells at the vegetal pole results in a noticeable thickening of epithelium termed the vegetal plate [18]. During gastrulation, the vegetal plate invaginates to produce the mesoderm and endoderm of the larva, leaving the remaining animal epithelium as ciliated ectoderm [18]. At this stage, no obvious morphological differences in ectodermal cell type have been observed, but we nonetheless reveal here a remarkable complexity of regulatory states within the ectoderm (summarized in Figure 1).

Transcripts of sea star regulatory genes are localized throughout the animal ectoderm in overlapping concentric domains along the AV axis. Some of these transcripts, such as those of zic, foxq2, $r x$ and $n k 2.1$, are found only in the animal-most ectoderm (Figures 2A$2 \mathrm{D})$. Of these genes, zic appears to be most closely localized to the animal pole, while expression of foxq 2 and $r x$ overlaps with $z i c$, yet extends further. Transcripts of six 3 and klf13 (Figures 2E and 2F) also are detected in the animal-most ectoderm of blastulae; however, they show a still broader distribution. Although there is no clear cell morphology that demarcates the boundary between the animal ectoderm and the vegetal plate endomesoderm, we observe a ring of $n k 1$ expression above the vegetal plate (Figure $2 \mathrm{G}$ ) that partially overlaps with endodermally localized gatae transcripts (Figure $2 \mathrm{H}$ ). The $n k 1$ expression domain therefore likely marks the vegetalmost ectoderm of the blastula. foxj 1 and pea3 (Figures 2I and 2J) are expressed throughout the ectoderm.

Taken together, the spatial expression of these regulatory genes demonstrates that the ectoderm of the sea star blastula is patterned along the AV axis in at least five nested concentric domains (summarized in Figure 2K).

\section{Regulatory gene expression within the ectoderm of the ciliary bands and animal pole domain}

During gastrulation, the ectoderm appears to undergo very little morphological change other than the coalescence of cilia within two bands: a preoral ciliary band that loops above the opening of the mouth and a postoral 
(a)

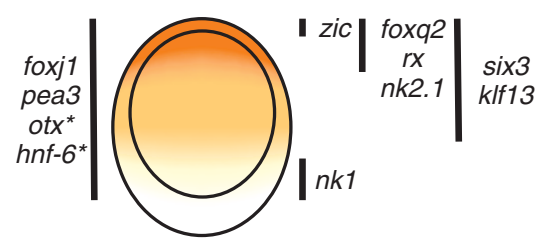

(d)

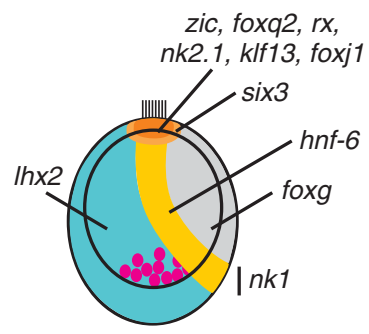

(b)

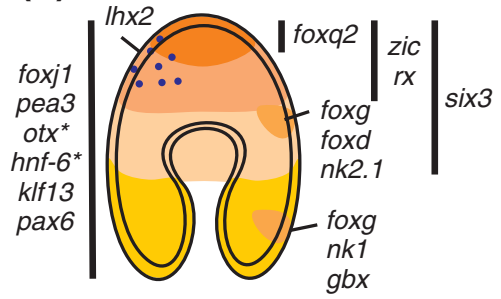

(e)

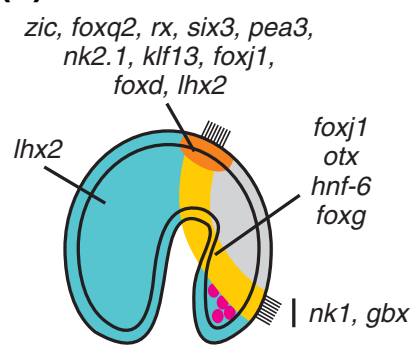

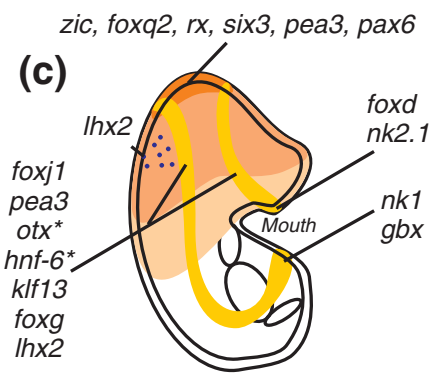

(f)

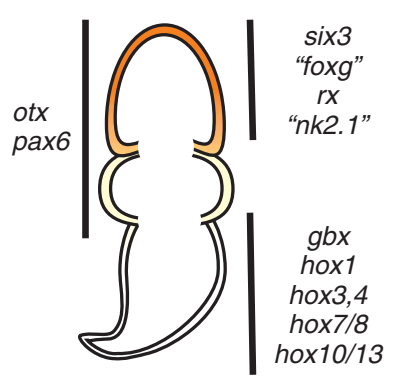

(g)

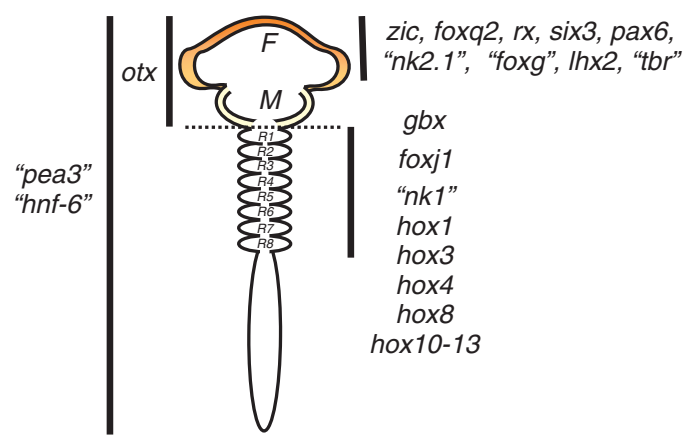

Figure 1 Comparison of orthologous neuroectodermal gene expression domains among the deuterostomes. (A-E) Indirectly developing echinoderms. (F) Directly developing hemichordate. (G) Generalized vertebrate. Sea stars (Figures 1A-1C) and sea urchins (Figures 1D and 1E) are viewed laterally; animal pole is up and oral side is right. Figures $1 \mathrm{~F}$ and $1 \mathrm{G}$ are dorsal views; anterior is up. Genes are listed beside their cognate expression domains. Vertical bars in Figures 1A, 1B, 1F and 1G approximate domain boundaries. The orange to yellow gradient in Figures 1A, 1F and $1 \mathrm{G}$ reflects a general conservation of anterior (animal)-most axial patterning among the three phyla. (A) Nested, concentric expression domains pattern the animal-vegetal (AV) axis of blastulae; asterisks denote previously reported expression [18,19]. (B) Concentric domains of zic, foxq2, rx and six3 persist in gastrulae (orange to peach gradient); additional oral (for example, foxg, foxd and gbx; light orange) and aboral (for example, Ihx2; purple) domains are evident. Genes (left) are broadly expressed. (C) Expression in larval animal pole domain (orange to peach) and/or ciliary bands (gold). (D) Sea urchin animal pole (orange and light orange), ciliary band (gold), aboral ectoderm (turquoise) and oral ectoderm (foxg; gray) are molecularly distinct territories in blastulae. (E) Expression is maintained in gastrulae animal pole (orange) and ciliary band (gold). (D and E) Pink circles represent skeletogenic mesoderm. See references [20,22,23,41-49]. (F) Orthologs expressed in hemichordate anterior, middle and posterior body segments show corresponding expression in the vertebrate forebrain, midbrain and hindbrain, respectively; data are summarized from Lowe et al. [13]. (G) Expression in generalized vertebrate centralized nervous system. F, forebrain; M, midbrain; R1-R8, rhombomeres of hindbrain. zic [50]; pea3 [51]; hnf-6/onecut [52]; and tbr [53]; foxj1 [54]; hox genes [55]. See references [24] and [26-32]. Echinoderm gene names (quotations) are substituted for simplicity in Figures $1 \mathrm{~F}$ and $1 \mathrm{G}$.

ciliary band that loops below it and around the aboral surface at the "back" of the embryo (Figure 3A) $[7,18]$. Transcripts of several genes that were distributed broadly throughout the ectoderm prior to gastrulation are later expressed within the ectoderm of the ciliary bands of the larva following gastrulation (for example, foxj 1 and $k l f 13$ in Figures 3B-3E, pea 3 as summarized in Figure 1 and as previously reported for otx and hnf-6/onecut expression $[18,19])$. At present, it is unclear if these patterns of expression reflect a migration of ectodermal cells to the 


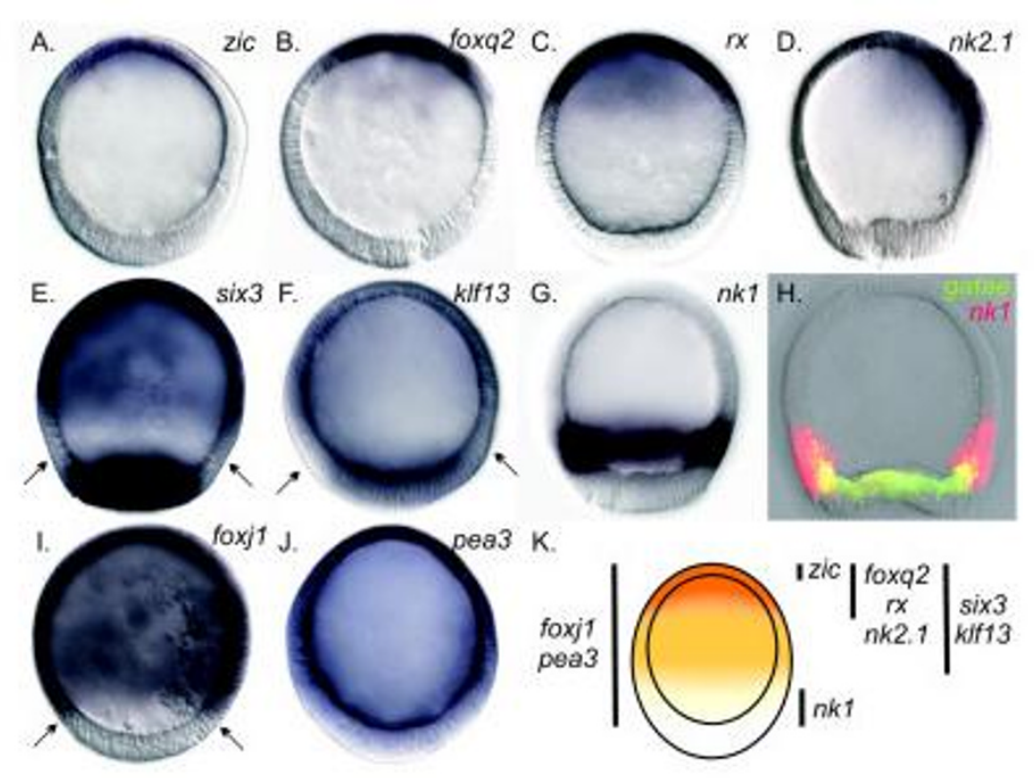

Figure 2 Nested concentric expression domains pattern the axial ectoderm of sea star, $P$. miniata, blastulae. Embryos are oriented with the animal pole up. (A-G) Whole mount in situ hybridization (WMISH). (A) zic, (B) foxq2, (C) $r x$, and (D) $n k 2.1$ expression is restricted to the animal-most ectoderm. Transcripts of (E) six3 and (F) klf13 are detected in the ectoderm and in the vegetal plate endomesoderm. Arrows in (E) and $\mathbf{( F )}$ point to a clearing above the vegetal pole where no or few transcripts are detected. (G) $n k 1$ transcripts are localized to a ring above the vegetal pole. $\mathbf{( H )}$ The boundary between the vegetal-most ectoderm (nk1, red) and the endoderm (gatae, green) as visualized by fluorescence in situ hybridization (FISH). Colocalization is in yellow. (I and $\mathbf{J})$ WMISH. Transcripts of (I) foxj1 and (J) pea3 are detected throughout the entire ectoderm. pea3 is weakly detected in the vegetal plate endomesoderm. Arrows in Figure $1 \mathrm{~J}$ point to the limits of foxj1 expression. (K) Schematic shows the patterns described above as five nested domains of expression along the AV axis. For simplicity, nk2.1 is grouped here with the concentric domains of foxq2 and rx expression. Gene names are listed next to their cognate expression domains. Vertical bars approximate the expression boundaries of associated genes. The color gradient spans the animal (orange) to vegetal (yellow) limits of the ectoderm.

sites of the future ciliary bands or if there is another patterning mechanism that restricts the earlier broad expression. Other transcripts are first detected at this stage within the ectoderm on the oral side of the gastrula and then later within the ciliary bands (for example, transcripts of foxg, foxd and $g b x$; Figures $3 \mathrm{~F}$ and $3 \mathrm{G}, 3 \mathrm{H}$ and $3 \mathrm{I}$, and $3 \mathrm{~J}$ and $3 \mathrm{~K}$, respectively). A two-probe whole mount in situ hybridization (WMISH) of foxg and $l h x 2$, a gene localized to the aboral ectoderm, further highlights the oral side restriction of foxg transcripts in the gastrula (Figure 3).

The expression patterns at this later stage also show that the regulatory state of the early larval ciliary bands is heterogeneous, for example, $n k 2.1$ and foxd are expressed in part of the preoral ciliary band directly above the mouth (Figures 3G and 3I, respectively), while $g b x$ and $n k 1$ are localized to part of the postoral ciliary band below the mouth (Figures $3 \mathrm{~K}$ and 3L, respectively). Therefore, while the regulatory state of the ciliary band ectoderm can be defined by a suite of transcription factors (that is, klf13, foxj1, pea3, foxg, otx and hnf-6/one$c u t$ ), they are further subdivided into pre- and postoral regions on the basis of the localization of foxd, $n k 2.1$, $n k 1$ and $g b x$.
Other transcripts that we detected within the ectoderm of the blastula remained within the animal ectoderm as gastrulation proceeded in what we define here as the animal pole domain. Unlike sea urchins, the sea star, P. miniata, does not appear to have a morphologically distinct animal pole domain at this stage. Transcripts of foxq2, pax6 and pea3 (Figures 4A-4C) tightly localize to the animal pole ectoderm, although their vegetal boundaries do not exactly coincide. Transcripts of $z i c, r x$ and six 3 are expressed within the animal pole domain as well, but even more vegetally throughout the animal ectoderm (Figures 4D-4F). The vegetal boundary of the animal pole domain therefore is not clearly defined by regulatory gene expression. The preoral and postoral ciliary bands run through the sea star animal pole domain as demonstrated by a two-probe fluorescence in situ hybridization (FISH) using the ciliary band marker, foxg, and the animal pole domain gene, pax6 (Figure 4G). Thus, despite its lack of morphological regionalization, the animal pole has a distinct regulatory state, as defined by foxq2, pax6, pea3, zic, $r x$ and six 3 expression, suggesting that it is a unique territory within the sea star. It is not yet clear whether these genes remain expressed in all cells of the sea star animal pole 
(a)

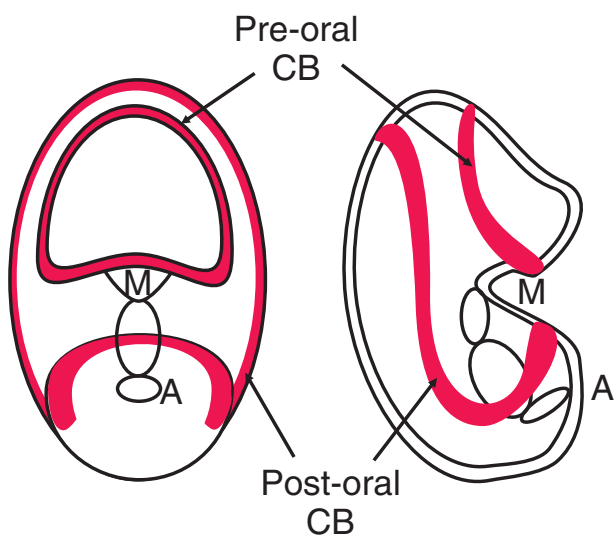

CB
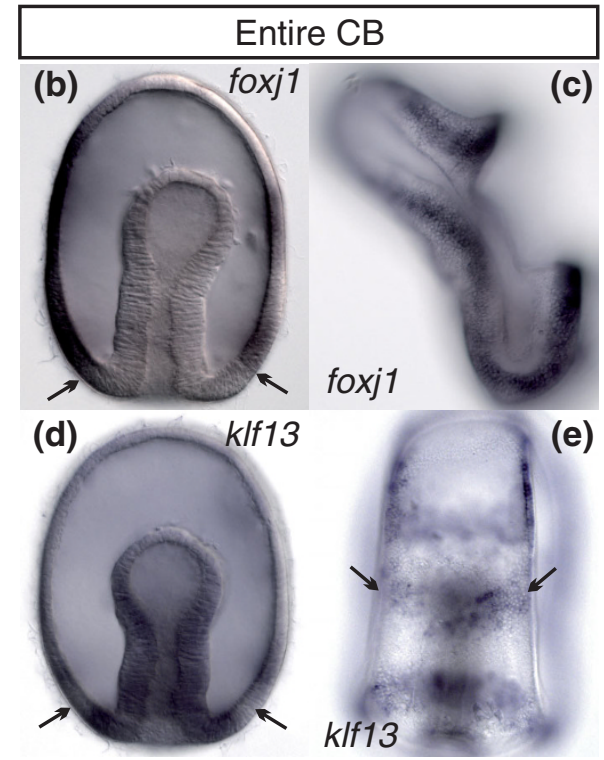

(f)
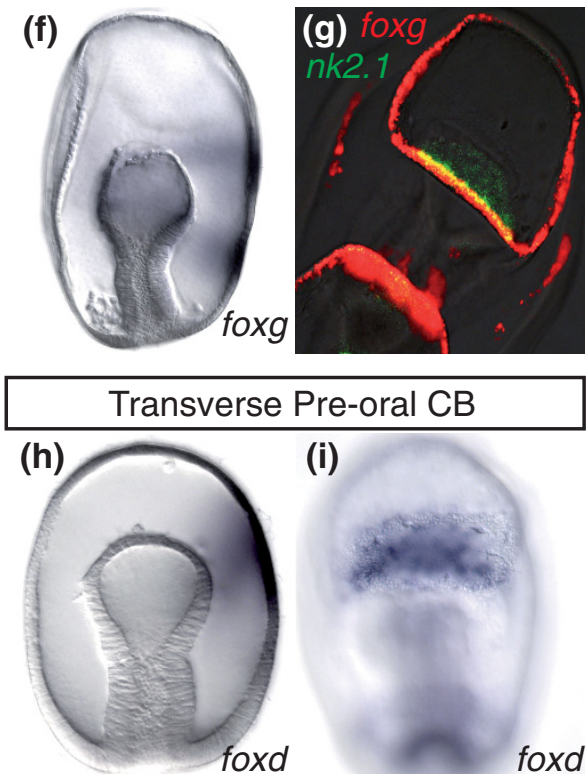

(i)

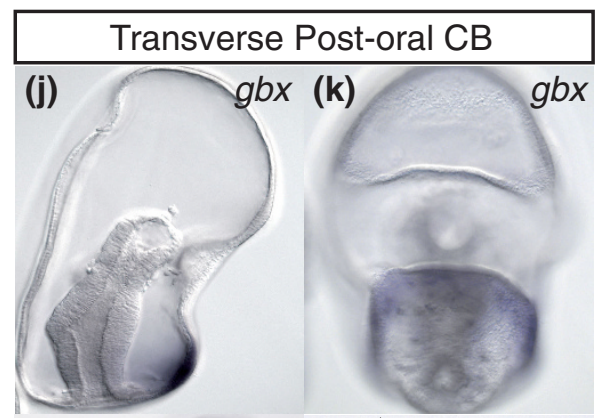

(I)

(m)

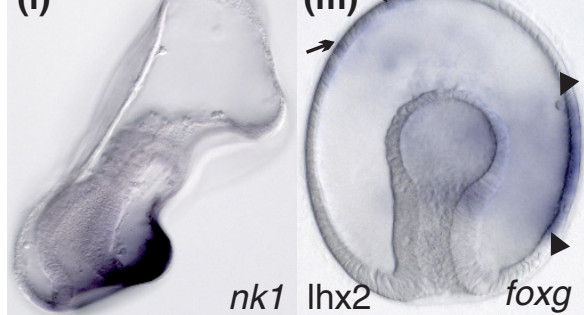

Figure 3 Heterogeneous regulatory patterning of the larval ciliary bands as visualized by WMISH. (A) Schematic describes the position of the two larval ciliary bands (red) from oral (left) and lateral (right) views. A, anus; CB, ciliary band; M, mouth. (B-F) WMISH. Expression of (B and C) foxj1 and (D and E) klf13 is initially broad throughout (B and $\mathbf{D})$ the ectoderm of gastrulae, then later is restricted to (C and $\mathbf{E})$ the larval ciliary bands. Arrows in Figure 3B show the vegetal limits of foxj1 expression. Arrows in Figure 3D point to a clearing above the vegetal pole where transcripts of klf13 were detected. Klf13 transcripts are additionally detected in an ectodermal territory near the mouth (arrows in Figure 3E). (F) foxg is first expressed within two ectodermal domains on the oral side of gastrulae. (G) FISH of $n k 2.1$ (green) and ciliary band marker foxg (red) highlights $n k 2.1$ expression in only the transverse preoral ciliary band. Colocalization is shown in yellow. (H-M) WMISH. foxd is expressed within a single domain in $\mathbf{( H )}$ the oral side ectoderm of gastrulae and $\mathbf{( I )}$ in the transverse, preoral larval ciliary band. gbx is expressed in one domain in (J) the oral side ectoderm in gastrulae and in (K) the transverse postoral larval ciliary band. (L) $n k 1$ is expressed in the transverse postoral ciliary band in the larva. (M) A two-probe WMISH shows Ihx2 expression in a spotted pattern in the aboral ectoderm (arrows, left) opposite of foxg expression (arrowheads, right). Embryos are oriented with the animal pole up and laterally, except in Figures $3 \mathrm{E}, 3 \mathrm{G}$, 3l and 3K, which are oral views. In lateral views, the oral side is to the right. 


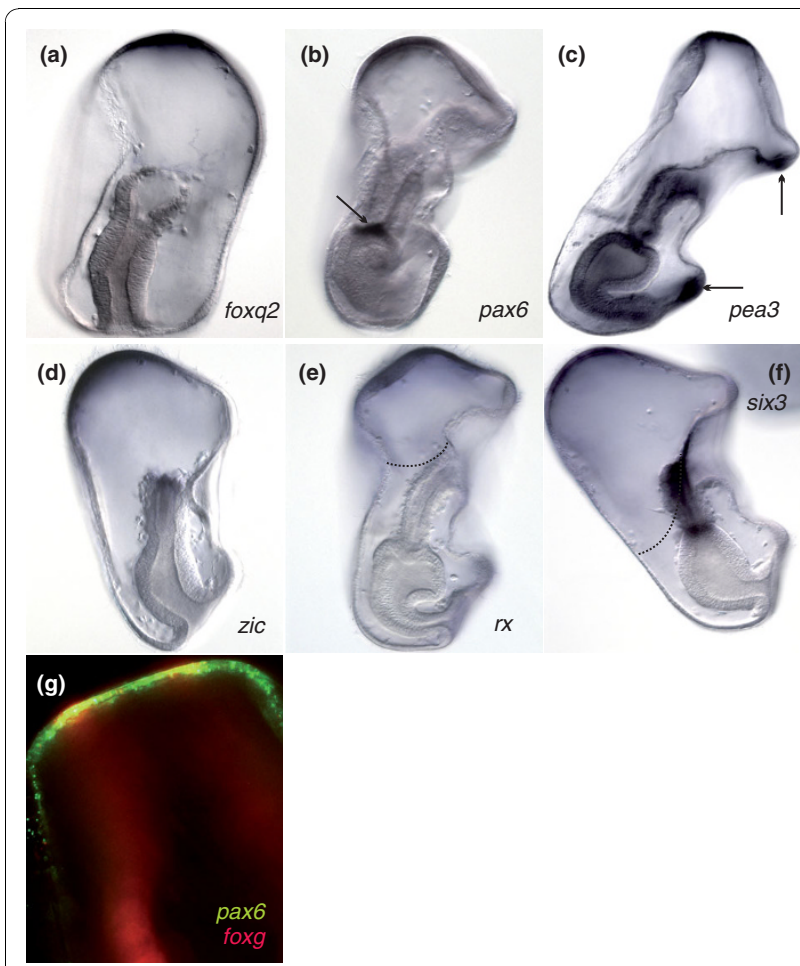

Figure 4 Gene expression molecularly defines the animal pole domain in the sea star. Embryos are shown laterally, with the animal pole up and oral side to the right. (A-F) WMISH. Expression of (A) foxq2, (B) pax6, (C) pea3, (D) zic, (E) $r x$ and (F) six3 within the apical-most ectoderm defines the animal pole domain within late gastrulae (Figures 4A, 4D and 4F) and early larvae (Figures 4B, 4C and $4 \mathrm{E}$ ). The vegetal limits of this domain are variable (see dotted lines in Figures 4E and 4F). Transcripts of pea3 additionally localize within the ectoderm of the larval ciliary bands (arrows in Figure 4C). pax6 expression in mesodermally derived coelom (arrow in Figure 4B). (G) FISH demonstrates that the ciliary bands, as marked by foxg (red), run through the ectoderm of the animal pole domain, as marked by pax6 (green). Colocalization is shown in yellow.

domain during later stages of larval development or if expression becomes refined to only subsets of cells within this domain.

\section{Comparisons of ectodermal patterning between sea urchin and sea star embryos}

At first inspection, the expression patterns of many genes appear markedly different in the earlier blastula stages of sea urchin and sea star embryos. The later restrictions within the animal pole or ciliary bands are, with some exceptions, more similar (Figure 1). We suggest that the sea urchin embryo may simply undergo a relatively more rapid specification of these territories, with an associated loss of intermediate domains that we observe in the sea star. Indeed, a careful examination of expression patterns in sea urchin has recently shown that the apical plate in sea urchin consists of at least two regulatory domains: an inner animal pole domain flanked by a ring of six3 expression [20]. These two domains in the sea urchin hatched blastula may therefore represent a more apically compressed version of the nested, concentric regulatory domains found in the sea star blastula.

Some of the patterning differences between sea urchin and sea star ectoderm also seem to account for the differences observed in the localization of the pan-neuronal marker, synaptotagmin-B [21]. For example, similar to the patterns of gene expression that we describe here, synaptotagmin-B is detected broadly throughout the ectoderm of the sea star gastrula, but in the larva it is found primarily in neurons associated with the ciliary bands and animal pole [9]. In the sea urchin, however, synaptotagmin-B is already localized to the animal pole domain and the presumptive ciliary band by the gastrula stage [9].

Although expression of many of the genes within the ciliary bands of the sea star appears conserved in the sea urchin, $n k 2.1$ and foxd show clear differences in expression that may be associated with the evolutionary transition from a double looping of the ciliary band around the body of the sea star bipinnaria and hemichordate tornaria to a single looping of the ciliary band observed in sea urchins. This single ciliary band in the sea urchin develops at the junction between oral and aboral ectoderm. $n k 2.1$ and foxd are expressed in part of the preoral ciliary band of the oral hood of the sea star, while sea urchin orthologs of these are found in the animal plate ectoderm (compare Figures $1 \mathrm{C}-1 \mathrm{E}$ ). Interestingly, both of these genes in sea urchin appear enriched on the oral side of the embryos [22,23]. Thus, we speculate that the preoral ciliary band may have been compressed into the oral-side animal plate territory in sea urchins and that this region within the sea urchin may therefore constitute a different territory than the remaining animal plate.

\section{Conservation of anterior (animal)-most regulatory patterning with other deuterostomes}

Comparisons of the regulatory gene expression patterns that we observed in these indirectly developing sea star embryos with those known in directly developing bilaterians illuminate additional surprising patterns of conservation. We observe a general mapping of gene expression patterns along body axes (compare Figures $1 \mathrm{~A}-1 \mathrm{C}$ with Figures 1F-1G). For instance, in the sea star, foxq2, $r x$, pax6 and six 3 orthologs are apically expressed within the ectoderm. foxq 2 expression in the amphioxus, a basal chordate, is restricted to the anterior-most end of the embryo [24]. Orthologs of $r x$ and pax6 are expressed in the anterior-most neuroectoderm in the hemichordate Saccoglossus [13], and they also pattern the anteriorly localized eye primordium in vertebrates $[25,26]$. The 
Drosophila $r x$ ortholog is required for brain development [27]. Orthologs of six3 and otx are expressed in anterior neuroectoderm in members of all three deuterostome phyla $[13,28,29]$. The most vegetal ectoderm in sea stars is characterized by the presence of $n k 1$ and $g b x$ transcripts. In vertebrates, a $g b x$ ortholog establishes the midbrain-hindbrain boundary [30]. The zebrafish ortholog of $n k 1$, sax2, is expressed within the midbrain-hindbrain boundary as well, although its expression is not exclusive to this territory [31]. Expression of $n k 1$ and $g b x$ in sea stars, and possibly sea urchins, marks the vegetal (posterior)-most ectoderm.

There is some evidence of additional conservation between the DV and oral-aboral axes as well. The mouse ortholog of $n k 2.1$ ( $n k x 2.1)$ is involved in the formation of motor neurons in the ventral telencephalon [32]. Saccoglossus $n k 2.1$ orthologs also show a ventral bias in expression [13]. Furthermore, foxg plays a role in ventral forebrain development, while $l h x 2$ specifies dorsal telencephalic fates [32]. We similarly show that expression of sea star orthologs of foxg and $n k 2.1$ is restricted to the oral (ventral) ectoderm, while $l h x 2$ orthologs are expressed within the aboral (dorsal) ectoderm (Figure 1B).

While in these comparisons we do not intend to convey a tight homology in gene expression patterns across deuterostome phyla, we predict that similarities in the overall patterning are an ancestral innovation and perhaps evidence of maintenance of some elements of a developmental gene regulatory network (GRN) inherited from a common ancestor. Conservation, however, is not maintained for orthologs of genes expressed within regions posterior to the midbrain-hindbrain boundary in chordates and hemichordates as $n k 1$ marks the vegetalmost ectoderm. Also, the overlapping expression of hox gene orthologs needed to pattern the posterior of many embryos are found only later in echinoderm development within the mesoderm of the rudiment [33].

\section{Separation of "retinal" from "anterior neural" regulatory patterning}

Vertebrate orthologs of transcription factors such as pax6, six 3 and $r x$ play known roles in pattering and specifying anterior vertebrate sensory systems, most notably the eyes $[25,26,34]$. Furthermore, orthologs of pax6, the six gene family members and eya operate in a similar gene network for retinal determination in both vertebrates and Drosophila (as reviewed in [35]).

Having established that orthologs of many regulatory genes involved in anterior neural specification are also expressed within the anterior ectoderm of sea star embryos, we sought to determine if orthologs of transcription factors involved within the retinal determination network are also expressed within echinoderm embryos.
We have already shown that the sea star pax6 ortholog is expressed within the animal pole domain (Figure 4B), although it is not expressed within the ectoderm of the sea urchin embryo (Figure 5). Transcripts of both pax6 and eya in both sea urchins and sea stars, however, are detected in the mesoderm of midgastrulae and then more prominently in one mesodermally derived coelom in late gastrulae (Figures 5A-5D and Figures 5G-5L). While we were unable to obtain a six 1.2 ortholog from the sea star, this gene is expressed also within the mesodermal coelom in the sea urchin (Figures 5E and 5F).

In the sea urchin, expression of two members of the light-sensing rhodopsin family of G-coupled protein receptors, opsin 1 and opsin 4 , has been shown as early as 1 week [36]. We were unable to obtain sea star opsin sequences; however, we confirmed the expression of opsin1 and opsin4 1-week-old sea urchin larvae (Figures $5 \mathrm{M}$ and $5 \mathrm{~N}$ ). The morphology of the late larval sea urchin embryos makes it difficult to decipher the precise location of these transcripts within the embryo. We therefore sought to determine if opsins collocalize with eya, which we show is expressed likely within one or both coeloms (depending on developmental timing) in 1-week-old larvae (Figure 5O). Using a two-probe FISH, we observe that transcripts of opsin 1 colocalized with those of eya in 1-week-old sea urchin larvae (Figures 5P5R). Expression of retinal determination orthologs within the mesoderm of gastrulae and larvae allow for the possibility that these genes operate within a common GRN.

The tightly coupled GRNs for anterior neural and visual sensory structures that are found in vertebrates and also in invertebrates, such as Drosophila, therefore are spatially separated in echinoderms. The presence of gene transcripts of pax6, rx and six3, but not, for example, eya, within the animal ectoderm of sea star bipinnaria larva may indicate a partial retention of an ancestral retinal determination network that once operated within this embryonic territory. This might also explain the absence of apically localized rhabdomeric eyespots, which are characteristic of the indirectly developing tornaria larvae of some hemichordates but were likely lost in the echinoderm lineage [16].

\section{Conclusions}

\section{Inferring ancestral states}

The detailed expression analyses reported here support the hypothesis that indirectly developing planktonic echinoderm embryos likely utilize ancient regulatory mechanisms for various anterior neuroectodermal and/ or sensory developmental processes that are potentially conserved throughout the Bilateria. Compared to vertebrates and well-studied protostome model organisms such as Drosophila, however, echinoderm embryos separate the deployment of these subcircuits in space and/or 


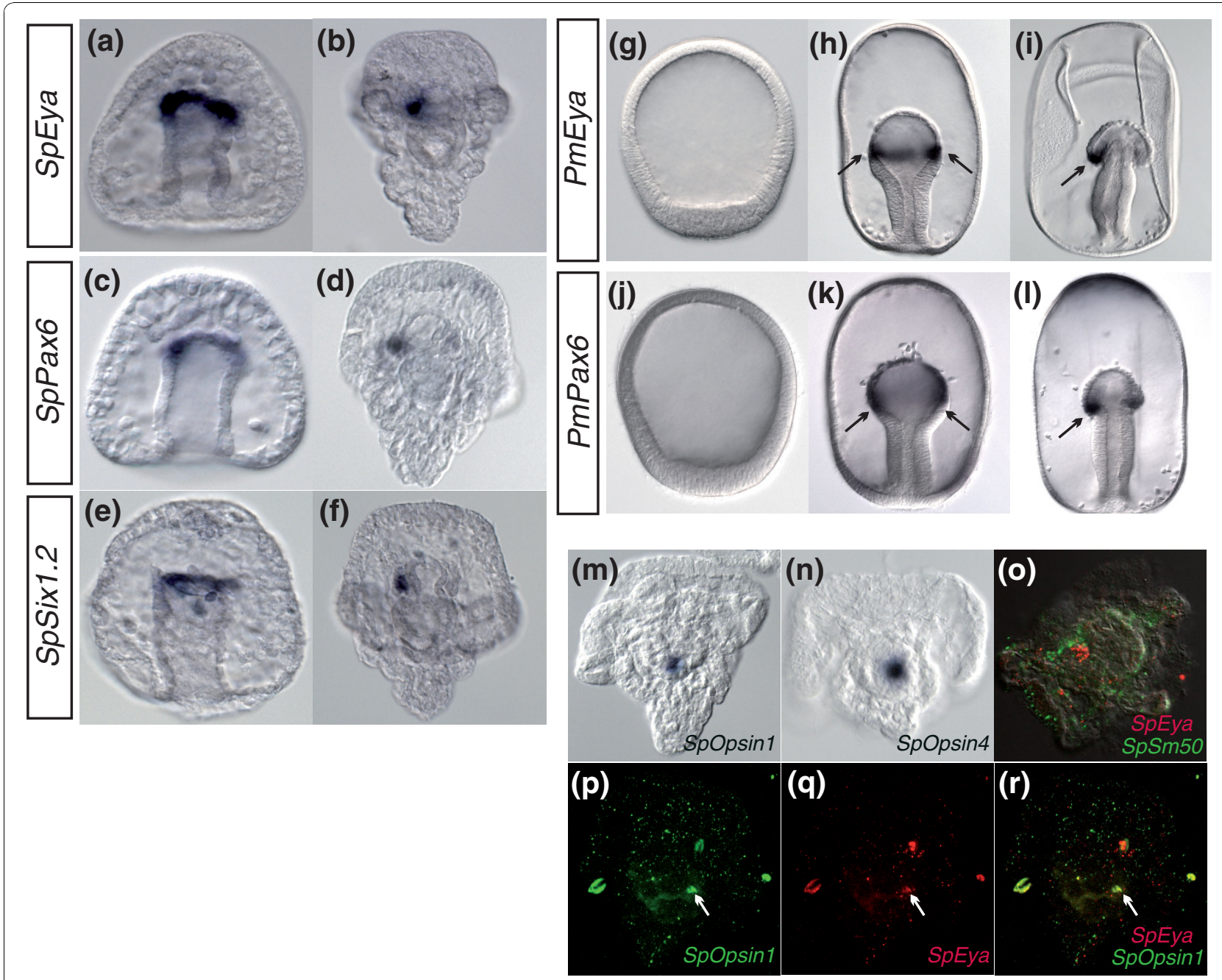

Figure 5 Retinal determination orthologs are expressed within sea urchin, (Strongylocentrotus purpuratus, Sp), and sea star mesoderm (A-N) WMISH. (A and B) SpEya, (C and D) SpPax6 and (E and F) SpSix 1.2 are expressed at the tip of the archenteron in gastrulae (Figures 5A, 5C and 5E) and in a mesodermally derived coelom in early pluteus larvae (Figures 5B, 5D and 5F). PmEya (Figures 5G-5I) and PmPax6 (Figures 5J-5L) expression is first detected in the mesoderm of the archenteron bulb in midgastrulae (Figures $5 \mathrm{H}$ and $5 \mathrm{~K}$; arrows) and then more prominently in a mesodermally derived coelom in late gastrulae (Figures $5 \mathrm{I}$ and 5L; arrow). PmPax6 transcripts are also found broadly throughout the ectoderm (Figure 5K), with more pronounced expression in the apical ectoderm (Figure 5L). SpOpsin 1 (Figure 5M) and SpOpsin4 (Figure 5N) are expressed in 1-week-old larvae. (O-R) FISH in 1-week-old larvae. SpEya (Figure 50) is shown relative to the skeletal marker SpSm50, which was used to orient the embryo. Figures 5P-5R show transcripts of SpOpsin1 (green) colocalizing with those of SpEya (red). Colocalization is shown in yellow. Arrows in Figures 5P, 5Q and 5R point to expression. Additional areas of expression within the 1-week-old larvae may be a result of nonspecific staining.

time. Thus, echinoderm embryos may have conserved sets of genetic regulatory relationships for "head/anterior brain" within the ectoderm of the early blastula and others for "retinal determination" within the mesodermal coelom of the gastrula.

Much of the difficulty in inferring the ancestral state of the deuterostomes and the mysteries of the origin of the phylum to which we belong arises from the complex life histories found within extant lineages [37]. Given the conservation of complex sensory and AP patterning between protostomes such as Drosophila and vertebrates, the parsimonious explanation is that ancestral developmental regulatory interactions, perhaps even entire GRN subcircuits, have been uncoupled along the lineage of echinoderms, possibly coincident with a simplification in early development. However, until a greater breath of taxa have resolved GRNs, we cannot know the flexibility with which modular subcircuits can be deployed during evolution of alternative body plans or if intercalation of GRN subcircuits occurs independently or coincident with an increase in complexity. 


\section{Methods}

\section{Sea star and sea urchin embryo culture and characterization of gene expression}

P. miniata embryos, previously named Asterina miniata, and Strongylocentrotus purpuratus embryos were cultured as described previously [18,38]. Partial gene sequences were obtained via screening a 3-day-old (lategastrula stage) P. miniata arrayed cDNA library using $S$. purpuratus sequence-specific probes and low stringency conditions as previously described [39]. Whole mount in situ hybridization (WMISH) was performed as described previously [18].

\section{Two-color FISH}

WMISH was performed essentially as described by Hinman et al. [18], with modifications to detect riboprobes using fluorescence as described by Denkers et al. [40]. In brief, both digoxygenin (DIG) and 2,4-dinitrophenol (DNP) labeled riboprobes were used. Hybridized probes were detected using anti-DIG antibody (1:2,000; Roche: Indianapolis, IN, USA) and anti-DNP antibody (1:1,000; PerkinElmer: Chicago, IL, USA), both conjugated to horseradish peroxidase, and the Tyramide Signal Amplification (TSA) Plus Fluorescence Systems Kit (PerkinElmer). A CyIII- or fluorescein-labeled tyramide was deposited near the in situ riboprobe in a reaction catalyzed by horseradish peroxidase, allowing for fluorescence detection of DIG- and DNP-labeled riboprobes.

\section{Additional material}

Additional file 1: Table 1. List of sea star, P. miniata, orthologs and orthology of gene sequences.

\begin{abstract}
Acknowledgements
The authors thank Dr Daniel Brown, Brenna McCauley, Alys Cheatle and three anonymous reviewers for helpful comments on the manuscript. This work was partially supported by National Science Foundation grant 0844948 to VFH, and CKJ and MLM were funded by a Howard Hughes Medical Institute Undergraduate Education Research Grant.
\end{abstract}

\section{Authors' contributions}

VFH and KAY conceived of the study, participated in its design and drafted the manuscript. KAY cloned sea star orthologs and performed WMISH and FISH. CKJ cloned sea urchin orthologs and performed WMISH. MLM cloned sea star orthologs and performed WMISH and FISH. All authors read and approved the final manuscript.

\section{Competing interests}

The authors declare that they have no competing interests.

Received: 5 August 2010 Accepted: 30 November 2010 Published: 30 November 2010

\section{References}

1. Bromham LD, Degnan BM: Hemichordates and deuterostome evolution: robust molecular phylogenetic support for a hemichordate + echinoderm clade. Evol Dev 1999, 1:166-171.
2. Castresana J, Feldmaier-Fuchs G, Yokobori S, Satoh N, Paabo S: The mitochondrial genome of the hemichordate Balanoglossus carnosus and the evolution of deuterostome mitochondria. Genetics 1998, 150:1115-1123.

3. Cameron CB, Garey JR, Swalla BJ: Evolution of the chordate body plan: new insights from phylogenetic analyses of deuterostome phyla. Proc Natl Acad Sci USA 2000, 97:4469-4474

4. Turbeville JM, Schulz JR, Raff RA: Deuterostome phylogeny and the sister group of the chordates: evidence from molecules and morphology. $\mathrm{Mol}$ Biol Evol 1994, 11:648-655.

5. Bourlat SJ, Juliusdottir T, Lowe CJ, Freeman R, Aronowicz J, Kirschner M, Lander ES, Thorndyke M, Nakano H, Kohn AB, Heyland A, Moroz LL, Copley RR, Telford MJ: Deuterostome phylogeny reveals monophyletic chordates and the new phylum Xenoturbellida. Nature 2006, 444:85-88.

6. Byrne M, Nakajima Y, Chee FC, Burke RD: Apical organs in echinoderm larvae: insights into larval evolution in the Ambulacraria. Evol Dev 2007, 9:432-445

7. Hyman LH: The Invertebrates: Echinodermata. New York: McGraw-Hill; 1955.

8. Hyman LH: The Invertebrates: Smaller Coelomate Groups. New York: McGraw-Hill; 1959.

9. Nakajima $Y$, Kaneko H, Murray G, Burke RD: Divergent patterns of neural development in larval echinoids and asteroids. Evol Dev 2004, 6:95-104.

10. Garstang W: Preliminary note on a new theory of the phylogeny of the Chordata. Zool Anz 1894, 17:122-125.

11. Lacalli TC: Apical organs, epithelial domains, and the origin of the chordate central nervous system. Am Zool 1994, 34:533-541.

12. Nielsen C: Origin of the chordate central nervous system - and the origin of chordates. Dev Genes Evol 1999, 209:198-205.

13. Lowe CJ, Wu M, Salic A, Evans L, Lander E, Stange-Thomann N, Gruber CE, Gerhart J, Kirschner M: Anteroposterior patterning in hemichordates and the origins of the chordate nervous system. Cell 2003, 113:853-865.

14. Lowe CJ, Terasaki M, Wu M, Freeman RM Jr, Runft L, Kwan K, Haigo S, Aronowicz J, Lander E, Gruber C, Smith M, Kirschner M, Gerhart J: Dorsoventral patterning in hemichordates: insights into early chordate evolution. PLOS Biol 2006, 4:e291.

15. Lacalli TC: Protochordate body plan and the evolutionary role of larvae: old controversies resolved? Can J Zool 2005, 83:216-224.

16. Brown FD, Prendergast A, Swalla BJ: Man is but a worm: chordate origins. Genesis 2008, 46:605-613.

17. Arendt D, Denes AS, Jekely G, Tessmar-Raible K: The evolution of nervous system centralization. Philos Trans R Soc Lond B Biol Sci 2008, 363:1523-1528.

18. Hinman VF, Nguyen AT, Davidson EH: Expression and function of a starfish Otx ortholog, AmOtx: a conserved role for Otx proteins in endoderm development that predates divergence of the eleutherozoa. Mech Dev 2003, 120:1165-1176.

19. Otim O, Hinman VF, Davidson EH: Expression of AmHNF6, a sea star orthologue of a transcription factor with multiple distinct roles in sea urchin development. Gene Expr Patterns 2005, 5:381-386.

20. Wei Z, Yaguchi J, Yaguchi S, Angerer RC, Angerer LM: The sea urchin animal pole domain is a Six3-dependent neurogenic patterning center. Development 2009, 136:1179-1189.

21. Burke RD, Osborne L, Wang D, Murabe N, Yaguchi S, Nakajima Y: Neuronspecific expression of a synaptatogmin gene in the sea urchin Strongylocentrotus purpuratus. J Comp Neurol 2006, 496:244-251.

22. Tu Q, Brown CT, Davidson EH, Oliveri P: Sea urchin Forkhead gene family: phylogeny and embryonic expression. Dev Biol 2006, 300:49-62.

23. Takacs CM, Amore G, Oliveri P, Poustka AJ, Wang D, Burke RD, Peterson KJ: Expression of an NK2 homeodomain gene in the apical ectoderm defines a new territory in the early sea urchin embryo. Dev Biol 2004, 269:152-164

24. Yu JK, Holland ND, Holland LZ: AmphiFoxQ2, a novel winged helix/ forkhead gene, exclusively marks the anterior end of the amphioxus embryo. Dev Genes Evol 2003, 213:102-105.

25. Bailey TJ, El-Hodiri H, Zhang L, Shah R, Mathers PH, Jamrich M: Regulation of vertebrate eye development by Rx genes. Int J Dev Biol 2004 48:761-770.

26. Pichaud F, Desplan C: Pax genes and eye organogenesis. Curr Opin Genet Dev 2002, 12:430-434. 
27. Davis RJ, Tavsanli BC, Dittrich C, Walldorf U, Mardon G: Drosophila retinal homeobox (drx) is not required for establishment of the visual system, but is required for brain and clypeus development. Dev Biol 2003, 259:272-287.

28. Simeone A, Acampora D, Mallamaci A, Stornaiuolo A, D'Apice MR, Nigro V, Boncinelli $\mathrm{E}$ : A vertebrate gene related to orthodenticle contains a homeodomain of the bicoid class and demarcates anterior neuroectoderm in the gastrulating mouse embryo. EMBO J 1993, 12:2735-2747.

29. Oliver G, Mailhos A, Wehr R, Copeland NG, Jenkins NA, Gruss P: Six3, a murine homologue of the sine oculis gene, demarcates the most anterior border of the developing neural plate and is expressed during eye development. Development 1995, 121:4045-4055.

30. Rhinn M, Brand M: The midbrain-hindbrain boundary organizer. Curr Opin Neurobiol 2001, 11:34-42.

31. Bae YK, Shimizu T, Muraoka O, Yabe T, Hirata T, Nojima H, Hirano T, Hibi M: Expression of sax1/nkx1.2 and sax2/nkx1.1 in zebrafish. Gene Expr Patterns 2004, 4:481-486.

32. Hebert JM, Fishell G: The genetics of early telencephalon patterning: some assembly required. Nat Rev Neurosci 2008, 9:678-685.

33. Arenas-Mena C, Cameron AR, Davidson EH: Spatial expression of Hox cluster genes in the ontogeny of a sea urchin. Development 2000, 127:4631-4643.

34. Carl M, Loosli F, Wittbrodt J: Six3 inactivation reveals its essential role for the formation and patterning of the vertebrate eye. Development 2002, 129:4057-4063.

35. Wawersik S, Maas RL: Vertebrate eye development as modeled in Drosophila. Hum Mol Genet 2000, 9:917-925.

36. Raible F, Tessmar-Raible K, Arboleda E, Kaller T, Bork P, Arendt D, Arnone MI: Opsins and clusters of sensory G-protein-coupled receptors in the sea urchin genome. Dev Biol 2006, 300:461-475.

37. Swalla BJ: Building divergent body plans with similar genetic pathways. Heredity 2006, 97:235-243.

38. Ettensohn CA, Wessel GM, Wray GA: Development of Sea Urchins, Ascidians, and Other Invertebrate Deuterostomes: Experimental Approaches. San Diego: Elsevier Academic Press; 2004

39. Hinman VF, Nguyen AT, Cameron RA, Davidson EH: Developmental gene regulatory network architecture across 500 million years of echinoderm evolution. Proc Natl Acad Sci USA 2003, 100:13356-13361.

40. Denkers N, Garcia-Villalba P, Rodesch CK, Nielson KR, Mauch TJ: FISHing for chick genes: triple-label whole-mount fluorescence in situ hybridization detects simultaneous and overlapping gene expression in avian embryos. Dev Dyn 2004, 229:651-657.

41. Materna SC, Howard-Ashby M, Gray RF, Davidson EH: The $\mathrm{C}_{2} \mathrm{H}_{2}$ zinc finger genes of Strongylocentrotus purpuratus and their expression in embryonic development. Dev Biol 2006, 300:108-120.

42. Burke RD, Angerer LM, Elphick MR, Humphrey GW, Yaguchi S, Kiyama T, Liang S, Mu X, Agca C, Klein WH, Brandhorst BP, Rowe M, Wilson K, Churcher AM, Taylor JS, Chen N, Murray G, Wang D, Mellott D, Olinski R, Hallbook F, Thorndyke MC: A genomic view of the sea urchin nervous system. Dev Biol 2006, 300:434-460.

43. Rizzo F, Fernandez-Serra M, Squarzoni P, Archimandritis A, Arnone Ml: Identification and developmental expression of the ets gene family in the sea urchin (Strongylocentrotus purpuratus). Dev Biol 2006, 300:35-48.

44. Howard-Ashby M, Materna SC, Brown CT, Chen L, Cameron RA, Davidson EH: Identification and characterization of homeobox transcription factors genes in Strongylocentrotus purpuratus, and their expression in embryonic development. Dev Biol 2006, 300:74-89.

45. Su YH, Li E, Geiss GK, Longabaugh WJ, Kramer A, Davidson EH: A perturbation model of the gene regulatory network for oral and aboral ectoderm specification in the sea urchin embryo. Dev Biol 2010, 329:410-21.

46. Otim O, Amore G, Minokawa T, McClay DR, Davidson EH: SpHnf6, a transcription factor that executes multiple functions in sea urchin embryogenesis. Dev Biol 2004, 273:226-243.

47. Poustka AJ, Kuhn A, Radosavljevic V, Wellenreuther R, Lehrach $H$, Panopoulou G: On the origin of the chordate central nervous system: expression of onecut in the sea urchin embryo. Evol Dev 2004, 6:227-236.

48. Minokawa T, Rast JP, Arenas-Mena C, Franco CB, Davidson EH: Expression patterns of four different regulatory genes that function during sea urchin development. Gene Expr Patterns 2004, 4:449-456.
49. Yuh CH, Brown CT, Livi CB, Rowen L, Clarke PJC, Davidson EH: Patchy interspecific sequence similarities efficiently identify positive cisregulatory elements in the sea urchin. Dev Biol 2002, 246:148-161.

50. Aruga J: The role of Zic genes in neural development. Mol Cell Neurosci 2004, 26:205-221.

51. Munchberg SR, Ober EA, Steinbeisser H: Expression of the Ets transcription factors erm and pea3 in early zebrafish development. Mech Dev 1999, 88:233-236.

52. Hong SK, Kim CH, Yoo KW, Kim HS, Kudoh T, Dawid IB, Huh TL: Isolation and expression of a novel neuron-specific onecut homeobox gene in zebrafish. Mech Dev 2002, 112:199-202.

53. Bulfone A, Smiga SM, Shimamura K, Peterson A, Puelles L, Rubenstein JL: Tbrain-1: a homolog of Brachyury whose expression defines molecularly distinct domains within the cerebral cortex. Neuron 1995, 15:63-78.

54. Aamar E, Dawid IB: Isolation and expression analysis of foxj1 and foxj1.2 in zebrafish embryos. Int J Dev Biol 2008, 52:985-991.

55. Tümpel $\mathrm{S}$, Wiedemann LM, Krumlauf R: Hox genes and segmentation of the vertebrate hindbrain. Curr Top Dev Biol 2009, 88:103-137.

doi:10.1186/1741-7007-8-143

Cite this article as: Yankura et al:: Uncoupling of complex regulatory patterning during evolution of larval development in echinoderms. BMC Biology 2010 8:143.

\section{Submit your next manuscript to BioMed Central and take full advantage of:}

- Convenient online submission

- Thorough peer review

- No space constraints or color figure charges

- Immediate publication on acceptance

- Inclusion in PubMed, CAS, Scopus and Google Scholar

- Research which is freely available for redistribution

Submit your manuscript at www.biomedcentral.com/submit
Biomed Central 